teachers of both geology and geography; and their value would be much greater to British students if the titles of the plates were repeated in English, for which there is abundant space on the explanatory sheets. The titles are short and necessarily technical, and their translation, unaided by context, would be often too difficult for the students to whom these photographs should be of most value.

J. W. G.

\section{THREE-COLOUR KINEMATOGRAPHY.}

I $N$ spite of the large amount of work which has 1 been done upon three-colour photography, it is doubtful whether the results obtained by the newer methods are in any way superior to those obtainable by the original three-colour projection process used by Maxwell in his classic lecture.

In three-colour photography, three negatives are taken, one through a red, a second through a green, and a third through a blue-violet filter. Having secured the negatives, we may then proceed to produce a three-colour photograph by one of two methods : either we may prepare black positives from our negatives and project the three positives by means of the three primary colours, or we may prepare from each negative a print in some substance such as bichromated gelatine, which can be dyed in the colour complementary to the taking filter. The superposition of these three-coloured positives will then give a coloured picture. It is found, however, in practice, that the first method, which is known as the additive process, gives results appreciably superior to the second or subtractive process. The cause of this lies in the fact that the dyes used for preparing the prints cannot completely absorb the colours which they are required to absorb, and, at the same time, completely transmit that portion of the spectrum which they should transmit. While the yellow dye is fairly satisfactory in this respect, and the red dye efficiently transmits red light, the red dye always fails to transmit sufficient blue light, and the light blue dye, used for the prints from the red negative, never transmits either sufficient blue light or sufficient green light, the deficiency in the transmission of green being usually very marked, and resulting in the reproduction of all dark green objects as browns, or at best, blacks.

For the first method or additive process it is, of course, necessary to use three separate projection sistems, and owing to the great loss of light through the filters these are advisedly supplied from three separate light sources. Unfortunately the unsteadiness of arcs makes it very difficult, if not impossible, to use three separate arcs, and consequently the best system is probably that arranged to use three large Nernst burners, the triple lantern being arranged so that the three projection systems stand vertically one above the other, and the three positives, printed on one plate, can be inserted together and focussed simultaneously, the adjustments for register being made once for all upon the lantern, so that subsequent slides register automatically. If an arc lamp is used, as in Mr. Ives's apparatus, only one lamp can be used, and the light from this must be divided into three by means of a system of reflectors, a procedure which unfortunately is frequently very wasteful of light and leads only to unsatisfactory results.

Attempts to apply triple projection methods to kinematography have frequently been made, but have not yet come before the public, except in the "Kinemacolor" method, which uses only two colours, abandoning the third, and so eliminating much complication. A two-colour method can, of course, at best be only a compromise, but it would seem at present to be the most satisfactory for kinematograph work.
In kinematograph work it is not necessary to project the colours to be combined simultaneously, as in the ordinary projection method. The colours can be successively projected, the observer combining them by persistence of vision. The three negatives are taken in turn on one film, a rotating shutter being arranged behind the lens of the camera carrying the three filters; but if the image is not to flicker strongly on the screen, it will be necessary for all three filters to be exposed within the ordinary period of exposure of a single kinematograph picture, which involves an exposure for each filter of about one-fiftieth of a second, a rate of work which is not only a great strain on the film and apparatus, but represents very brief exposures when it is remembered that the negatives must be taken through strongly absorbing red and green filters.

Attempts have been made to project the three images simultaneously by means of three separate optical systems, as in ordinary three-colour work, but the difficulties of register have been found very serious, although it is not at all clear why this should be so if both positive and negative sets of pictures are handled simultaneously on films of three times the normal width.

In the "Kinemacolor" method, the spectrum is divided into two, one taking-filter being orange-red and the other blue-green. In order to get a satisfactory rendering of greens, the latter taking-filter must be rather greener than a true blue-green filter would be, and in the resulting picture blue is little distinguishable from blue-green, while a yellow is rendered rather as an orange than a true yellow. The results are, however, surprisingly good considering the theoretical difficulties of the method and the extreme badness of ordinary two-colour work, and they will doubtless be even better when the promoters of the process have learned that above all things they must avoid in their pictures the two colours which they are using for projecting. These two colours are a pure red and a very bright blue-green, or minus-red colour, and in some of the pictures at present there is a tendency to use these two colours in the composition of the original scene, so that one gets little girls dressed in red blouses and blue-green skirts, which colours. the process renders with only too distressing fidelity. Considering the excellent way in which the process reproduces subdued colours, such as browns, there really seems no need for this. A much less serious: defect in the process, and one which is, of course, unavoidable, is that when anything moves rapidly it is projected as a series of coloured images, so that a horse's leg on the screen may appear as a series: of alternately red and blue-green legs.

There is no doubt, however, that kinemacolour is a success, and is a striking testimony to the good practical results which may sometimes be obtained from a theoretically inaccurate system.

In a recent patent it has been suggested that the successive and synchronous methods of projection might be combined, two projecting systems being used with two sets of pictures, one set representing only one-colour element, such, for instance, as red, while the other is composed alternately of pictures of the other two-colour elements, blue and green. A stationary red filter is used in front of one projecting lens, while the second film is projected through a rotating filter, part green and part violet. This would appear to be somewhat more complicated than the two-colour system, while the colour rendering should undoubtedly be superior; but the method does not appear to have, at present, reached the stage of practical use.

C. E. Kenneth Mees.

NO. 2 I9I, VOL. 87] 